\title{
Charged-particle interaction with liquids: Ripplon excitations
}

\author{
Nuria Barberan \\ Departamento de Estructura y Constituyentes de la Materia, Facultad de Fisica, Universidad de Barcelona, \\ Diagonal 647, E-08028 Barcelona, Spain \\ Rafael Garcia-Molina \\ Departamento de Fisica Aplicada, Facultad de Ciencias, Universidad de Murcia, E-30071 Murcia, Spain
}

Alberto Gras-Marti

Laboratori de Capes Fines $i$ Collisions Atómiques, Departmento de Fisica Aplicada, Facultat de Ciéncies, Universitat d'Alacant, Apartat 99, E-03080 Alacant, Spain

(Received 24 January 1989)

\begin{abstract}
We calculate the ripplon field contribution to the self-energy of an electron exterior to a liquid for planar and spherical geometries. We compare the full dielectric calculation of the electron-liquid interaction with the simpler alternative method consisting of integrating the electron-atom staticinduced-dipolar potential through the whole liquid volume. We obtain good agreement between both methods for a nonpolar liquid such as ${ }^{4} \mathrm{He}$ but differences up to $40 \%$ for a polar liquid such as water. We study the conditions under which the ripplon contribution to the self-energy is a perturbation. For an electron moving parallel to a planar liquid surface, we calculate the ripplon contribution to its stopping power. For this dynamical case, we conclude that the alternative method is a good approximation even for polar liquids.
\end{abstract}

\section{INTRODUCTION}

Long-range forces between charged particles and material surfaces are usually attractive with dissipative components and may result in the capture by the surface of the passing charge moving near it. The existence of electrons in surface states held near a liquid-helium surface has been verified experimentally, ${ }^{1,2}$ and the twodimensional electron lattice formed at low-temperature and high areal electron density has been extensively studied. ${ }^{3-7}$ Studies of free negatively charged water clusters of the form $\left(\mathrm{H}_{2} \mathrm{O}\right)_{n}{ }^{-}$have been reported, ${ }^{8-10}$ and the possibility of electron surface states on spherical water drops has been recently studied by Ballester and Antoniewicz. ${ }^{11}$ One may note also that aerosol transport and accretion may strongly depend on the charge state of the drops involved.

Because of the very strong repulsive short-range potential at the surface due to the exclusion principle, the surface-state wave function is usually considered not to penetrate inside the liquid. Nevertheless, the self-energy of the bound surface state is very sensitive to the interaction with ripplon excitations (surface shape oscillations). Variation of the electron mobility coming from electronripplon interaction and typical resonance effects from phonon-ripplon coupling has been studied. ${ }^{2,4,12}$ Ripplon excitations must also be included in order to obtain the elastic reflection coefficients for ${ }^{4} \mathrm{He}$ atoms hitting a liquid-helium surface. ${ }^{13}$

The method usually employed in the literature to study the particle-ripplon interaction is adequate for nonpolar liquids like helium and consists in the integration of the electron-helium atom potential through the helium volume to obtain the image potential, i.e.,

$$
V(\overline{\mathbf{r}})=-\frac{n \alpha e^{2}}{2} \int \frac{d \overline{\mathbf{r}}^{\prime}}{\left|\overline{\mathbf{r}}-\overline{\mathbf{r}}^{\prime}\right|^{4}},
$$

where $n$ and $\alpha$ are the atomic density and polarizability, respectively, and $e$ is the electronic charge. $V(\overline{\mathbf{r}})$ is a good approximation to the image potential because the induced electric dipole moment is small and screening effects are not very important. For polar liquids, however, a new coupling term must be invoked because of their atomic (or molecular) permanent dipole moment, inferred from the large value of their static dielectric constant. In this paper we perform a calculation of the image potential outside the dielectric liquid including the full charge liquid interaction within a dielectric response model. We follow the method used by Rahman and Maradudin ${ }^{14}$ in their calculation of the effect of surface roughness on the image potential and extend the work of Eguiluz et al., for planar surfaces ${ }^{6}$ and the work of Gras-Marti and Ritchie for spherical surfaces ${ }^{15}$ to polar liquids such as water.

There is an old discussion about the validity of using first-order perturbation theory for the electronic surfacestate ripplon interaction. This question was first raised by $\mathrm{Cole}^{16}$ and later clarified by Shikin and Monarkha, ${ }^{2}$ who realized that the use of the adequate boundary conditions at the interface guarantees the validity of the perturbation approximation for a planar liquid helium surface. We study again the validity of the perturbation approximation for polar liquids and for different geometries.

This paper is organized as follows. In Sec. II we obtain the interaction Hamiltonian for a point charge in front of a planar rippling surface. From that Hamiltonian we get 
the contribution to the self-energy of the point charge due to its interaction with the ripplon field. For helium and water, we compare the results obtained using this Hamiltonian with the ones obtained using Eq. (1). The spherical rippling surface case is treated in Sec. III. For a fixed reference axis, we calculate the contribution to the self-energy that comes from the charge-ripplon interaction for an external electron in the $z$ axis. The expressions of the coupling coefficients for an electron in an arbitrary position (which are needed for dynamical calculations) are given in the Appendix. Using the dipoleinduced potential and dielectric constant methods, we calculate in Sec. IV the stopping power for a swift electron moving external and parallel to a planar liquid surface of helium or water. Finally, some comments and conclusions are drawn in Sec. V.

\section{STATIC CHARGE RIPPLON INTERACTION: PLANAR LIQUID SURFACE}

We consider a static charged particle in front of a semi-infinite liquid bounded by a planar surface. The rippling of the surface is treated as a small perturbation to the flat surface and in all the calculations below we shall only keep first-order terms in that perturbation parameter. An incompressible liquid will be assumed in all that follows.

\section{A. Interaction Hamiltonian}

In the absence of the rippling perturbation, the liquid is taken to occupy the half space $z \leq 0$. We define $\bar{\rho}(x, y)$ as a two-dimensional vector parallel to the equilibrium planar liquid surface and $\zeta(\bar{\rho})$ denotes the amplitude of the surface deformation at $\bar{\rho}$ produced by the presence of the rippling perturbation [see Fig. 1(a)]. We introduce the two-dimensional Fourier transform of the surface displacement, $\zeta(\overline{\mathbf{q}})$, according to the equation

$$
\zeta(\overline{\boldsymbol{\rho}})=\Omega^{-1 / 2} \sum_{\overline{\mathbf{q}}} \zeta(\overline{\mathbf{q}}) e^{i \overline{\mathbf{q}} \cdot \bar{\rho}},
$$

which corresponds to an expansion of the surface displacement in ripplon normal modes (ripplons are the quanta of capillary waves at the surface of a liquid, see below). $\Omega$ is the normalization area. The polarization of the liquid is described by a dielectric constant $\epsilon$, which is assumed to be independent of position right up to the rippled liquid surface.

The scalar potential $\phi\left(\overline{\mathbf{r}}, \overline{\mathbf{r}}^{\prime}\right)$ created at $\overline{\mathbf{r}}$ by the presence of a point charge at $\overline{\mathbf{r}}^{\prime}$ outside the liquid is obtained from the Poisson equation and the appropriate boundary conditions at the deformed surface, i.e., the continuity of the scalar potential and the proper jump discontinuity of its normal derivative across this surface. From the knowledge of $\phi\left(\overline{\mathbf{r}}, \overline{\mathbf{r}}^{\prime}\right)$ we obtain the force that the polarized liquid exerts on an external electron at $\overline{\mathbf{r}}$ and after identifying the charge at $\overline{\mathbf{r}}^{\prime}$ and the electron at $\overline{\mathbf{r}}$, we calculate up to $O(\zeta)$ the work that must be done to bring the electron from infinity to the point $\overline{\mathbf{r}}=z \hat{\mathbf{z}}+\overline{\boldsymbol{\rho}}$ above the liquid surface. That gives the image potential energy, i.e.,
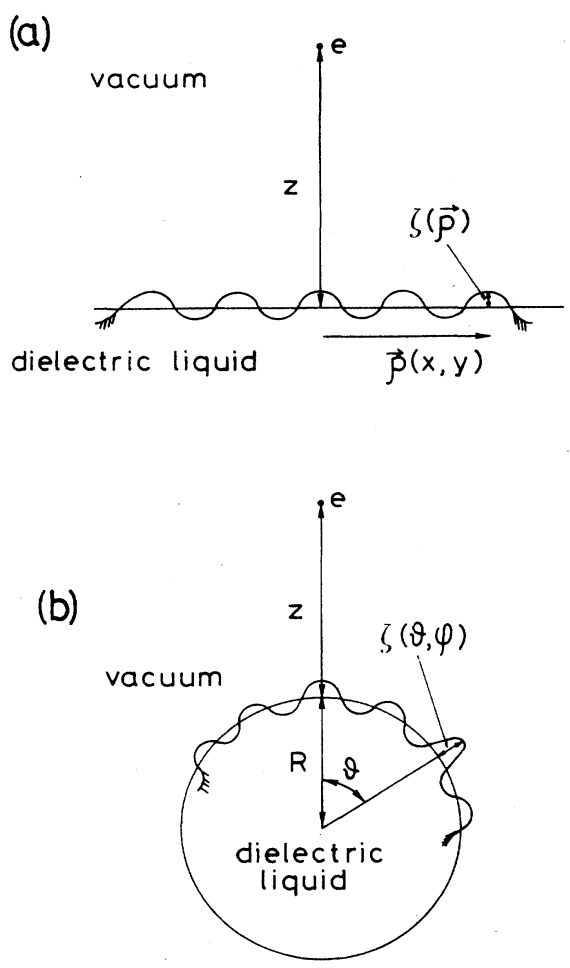

FIG. 1. Geometrical scheme used to describe the chargeripplon interaction: (a) planar rippling surface; (b) spherical rippling surface. The plane $x y$, where also the azimuthal angle $\varphi$ in (b) lies, is perpendicular to the $z$ axis. See text for definitions of the symbols.

the effective potential energy which enters the Hamiltonian that describes the system ${ }^{14,17,18}$

$$
\begin{array}{r}
U(\overline{\mathbf{r}})=-\frac{e^{2}}{4} \frac{\epsilon-1}{\epsilon+1} \frac{1}{z}\left(1+\frac{1}{2(\epsilon+1)} \int \frac{d^{2} q}{(2 n)^{2}} e^{i \overline{\mathbf{q}} \cdot \bar{\rho}} \zeta(\overline{\mathbf{q}}) q\right. \\
\times\left[2(\epsilon+1) K_{1}(q z)\right. \\
\left.\left.+(\epsilon-1) q z K_{0}(q z)\right]\right),
\end{array}
$$

where $K_{0}$ and $K_{1}$ are modified Bessel functions of the second kind. ${ }^{19}$ The first term in Eq. (3),

$$
U_{0}(\overline{\mathbf{r}})=-\frac{e^{2}}{4 z} \frac{\epsilon-1}{\epsilon+1},
$$

gives the interaction energy (the classical image potential) between an external charge and a flat dielectric surface. This term contains a residual contribution of the ripplon interaction ${ }^{6}$ which is made explicit if a Taylor series expansion around $\xi=z-\xi$ (the actual charge surface distance) is performed and the first-order term on $\zeta$ is retained. This is equivalent to using the appropriate boundary conditions of Shikin and Monarkha. ${ }^{2}$ This $\zeta$-dependent term together with the remaining two terms in Eq. (3) give the ripplon contribution to the image potential, 


$$
U_{\text {rip }}(\overline{\mathbf{r}})=\frac{\epsilon-1}{\epsilon+1} \frac{e^{2}}{4} \int \frac{d^{2} q}{(2 n)^{2}} e^{i \overline{\mathbf{q}} \cdot \bar{\rho}} \zeta(\overline{\mathbf{q}})\left[\frac{1}{\xi^{2}}-\frac{q}{2 \xi(\epsilon+1)}\left[(\epsilon-1) q \xi K_{0}(\xi q)+2(\epsilon+1) K_{1}(\xi q)\right]\right)
$$

When the ripplon field is quantized according to the usual canonical quantization procedure, the following relation for the Fourier coefficients is obtained: ${ }^{16}$

$$
\zeta(\overline{\mathbf{q}})=\left(\frac{\hbar q \Omega}{2 d w_{q}}\right)^{1 / 2}\left(b_{-\overline{\mathbf{q}}}^{\dagger}+b_{\overline{\mathbf{q}}}\right)
$$

where $d$ is the mass density of the liquid and $b_{\bar{q}}^{\dagger}$ and $b_{\bar{q}}$ are ripplon creation and annihilation operators. The ripplon frequency $w_{q}$ for a planar liquid surface appearing in Eq. (6) is given by the dispersion relation ${ }^{20}$

$$
w_{q}^{2}=g q+\frac{\sigma}{d} q^{3}
$$

where $g$ and $\sigma$ are, respectively, the gravity acceleration and the liquid surface tension.

Equations (5) - (7) lead us to the following result for the Hamiltonian describing the ripplon charge interaction:

$$
H^{1}=H_{1}+H_{2}+H_{3}
$$

with

$$
H_{i}=\sum_{\overline{\mathbf{q}}} \alpha_{i}(\overline{\mathbf{q}}, z)\left(b_{-\overline{\mathbf{q}}}^{\dagger}+b_{\overline{\mathbf{q}}}\right), i=1,2,3
$$

The coupling coefficients are given by

$$
\begin{aligned}
& \alpha_{1}(\overline{\mathbf{q}}, z)=-\frac{e^{2}}{4}\left(\frac{\epsilon-1}{\epsilon+1}\right)\left(\frac{\hbar q^{3}}{2 d \Omega w_{q}}\right)^{1 / 2} e^{i \overline{\mathbf{q}} \cdot \bar{\rho}} \frac{K_{1}(q z)}{z}, \\
& \alpha_{2}(\overline{\mathbf{q}}, z)=-\frac{e^{2}}{8}\left[\frac{\epsilon-1}{\epsilon+1}\right)^{2}\left[\frac{\hbar q^{5}}{2 d \Omega w_{q}}\right)^{1 / 2} e^{i \overline{\mathbf{q}} \cdot \bar{\rho}} K_{0}(q z), \\
& \alpha_{3}(\overline{\mathbf{q}}, z)=\frac{e^{2}}{4}\left(\frac{\epsilon-1}{\epsilon+1}\right)\left[\frac{\hbar q}{2 d \Omega w_{q}}\right]^{1 / 2} e^{i \overline{\mathbf{q}} \cdot \bar{\rho} \frac{1}{z^{2}}},
\end{aligned}
$$

where we express by $z$ the mean value of $\xi$.

For a nonpolar liquid the static dielectric constant is not very different from 1 ( $\epsilon=1.057$ for liquid helium) and a usual approximation is to neglect the second coupling coefficient, i.e., the term in $K_{0}$. This is justified because of the smallness of $\epsilon-1$, but we cannot neglect the third coupling coefficient since it becomes the dominant term for large distances from the planar surface. For polar liquids, $\epsilon$ is much bigger than 1 (that is the case of water with $\epsilon=78.1$ ) and one cannot neglect any of the coupling coefficients.

The total Hamiltonian for the point-charge plus ripplon field is then given by

$$
H=H_{0}+H^{\prime} \text {, }
$$

where $H_{0}$ is the Hamiltonian for free ripplons (15) and (16), which are excitations of boson character and therefore

$$
H_{0}=\sum_{\overline{\mathbf{q}}} \hbar w_{q}\left(b_{\overline{\mathbf{q}}}^{\dagger} b_{\overline{\mathrm{q}}}+\frac{1}{2}\right)
$$

\section{B. Self-energy}

The point-charge self-energy in the presence of the ripplon field can be easily calculated from the Hamiltonian given in Eq. (11). To find the system energy shift due to the electron ripplon interaction one makes use of a bilinear transformation that reduces the Hamiltonian given by Eq. (11) to the standard number operator form ${ }^{15,21}$ and the desired self-energy is the zero-point energy of the reduced Hamiltonian,

$$
\Delta E(z)=-\sum_{\overline{\mathrm{q}}}\left|\alpha_{1}+\alpha_{2}+\alpha_{3}\right|^{2} \frac{1}{\hbar w_{q}},
$$

where the terms appearing in the summation are given by

$\Delta E_{1}(z) \equiv-\sum_{\bar{q}} \frac{\left|\alpha_{1}\right|^{2}}{\hbar w_{q}}=-4 G v^{2} \int_{0}^{a} d t \frac{t^{3}}{1+\eta t^{2}} K_{1}^{2}(t)$,

$\Delta E_{2}(z) \equiv-\sum_{\overline{\mathrm{q}}} \frac{\left|\alpha_{2}\right|^{2}}{\hbar w_{q}}=-G v^{4} \int_{0}^{a} d t \frac{t^{5}}{1+\eta t^{2}} K_{0}^{2}(t)$,

$\Delta E_{3}(z) \equiv-\sum_{\overline{\mathrm{q}}} \frac{\left|\alpha_{3}\right|^{3}}{\hbar w_{q}}=-4 G v^{2} \int_{0}^{a} d t \frac{t}{1+\eta t^{2}}$,

$$
\begin{aligned}
\Delta E_{4}(z) & \equiv-2 \sum_{\overline{\mathrm{q}}} \frac{\operatorname{Re}\left(\alpha_{1} \alpha_{2}^{*}\right)}{\hbar w_{q}} \\
& =-4 G v^{3} \int_{0}^{a} d t \frac{t^{4}}{1+\eta t^{2}} K_{0}(t) K_{1}(t),
\end{aligned}
$$

$\Delta E_{5}(z) \equiv-2 \sum_{\overline{\mathrm{q}}} \frac{\operatorname{Re}\left(\alpha_{1} \alpha_{3}^{*}\right)}{\hbar w_{q}}=8 G v^{2} \int_{0}^{a} d t \frac{t^{2}}{1+\eta t^{2}} K_{1}(t)$,

$$
\Delta E_{6}(z) \equiv-2 \sum_{\overline{\mathrm{q}}} \frac{\operatorname{Re}\left(\alpha_{2} \alpha_{3}^{*}\right)}{\hbar w_{q}}=4 G v^{3} \int_{0}^{a} d t \frac{t^{3}}{1+\eta t^{2}} K_{0}(t)
$$

where

$$
G=\frac{e^{4}}{\left(256 \pi d g z^{6}\right)}, \quad v=\frac{(\epsilon-1)}{(\epsilon+1)}, \quad a=z q_{c},
$$


and where

$$
\eta=\frac{\sigma}{d g z^{2}}
$$

The upper limit of the integrals, $z q_{c}$, has the following physical meaning: The wavelength of the excited (virtual) ripplons cannot be smaller than a characteristic length for which the continuous description we are using for the liquid is not valid. The self-energy is not very sensitive to this upper limit when the smaller characteristic length we take is of the order of a few Bohr radii, corresponding to the mean separation between atoms or molecules in the liquid. $^{22}$ However, there is another wavelength cutoff criterion related to the ripplon energy which is competing with the one already mentioned. If we assume that in the static case the system is in thermal equilibrium, most of the thermally excited ripplons have energies of the order of $k_{B} T$, where $k_{B}$ is the Boltzmann constant and $T$ is the temperature, i.e., they have a long wavelengths. We choose the lowest of both cutoffs in each case.

In our calculations we use the following parameters: For liquid water at $0{ }^{\circ} \mathrm{C}, \sigma=72.8 \mathrm{dyn} / \mathrm{cm}, d=1 \mathrm{~g} / \mathrm{cm}^{3}$, $\epsilon=78.1$, and $q_{c}=2.1 \times 10^{8} \mathrm{~cm}^{-1}$, and, for liquid helium at $1 \mathrm{~K}, \sigma=0.35 \mathrm{dyn} / \mathrm{cm}, d=0.14 \mathrm{~g} / \mathrm{cm}^{3}, \epsilon=1.057$, and $q_{c}=1.9 \times 10^{7} \mathrm{~cm}^{-1}$.

In Fig. 2 we show numerical results for the self-energy as a function of the electron-surface mean distance $z$. We display also for comparison the classical image potential of the electron in front of a flat surface. In the helium case [Fig. 2(a)] the electron-ripplon contribution is always smaller than the image potential (less than 10\%). Therefore, the ripplon interaction is a perturbation of a welldefined electronic state (in the case of a surface state we should add the kinetic energy to the potential energy). This is in agreement with the findings of Shikin and Monarkha. ${ }^{2}$ However, in the case of water the perturbing condition is not fulfilled at short distances from the surface when, as it is the case in our calculation, the electron is represented by a static point charge. A full calculation of the quantum electronic wave function should be done to decide about the validity of the perturbation approximation in the region close to the surface.

We also compare the self-energies obtained from Eq. (13) and the one derived from Eq. (1) (removing the three $K_{0}$-dependent terms). We consider that the relation between the atomic polarizability and the dielectric constant is given by

$$
n \alpha=\frac{1}{2 \pi} \frac{\epsilon-1}{\epsilon+1}
$$

which is valid for planar geometries. For helium we obtain differences smaller than $1 \%$ between both methods, but in the case of water, the difference increases from $11 \%$ at 100 a.u. to $46 \%$ at 6 a.u. from the surface. As it is expected, the second method works worse for polar liquids because Eq. (1) does not include the full polarization of the dielectric and it is not taking into account the screening effects between the atoms or molecules in the liquid.
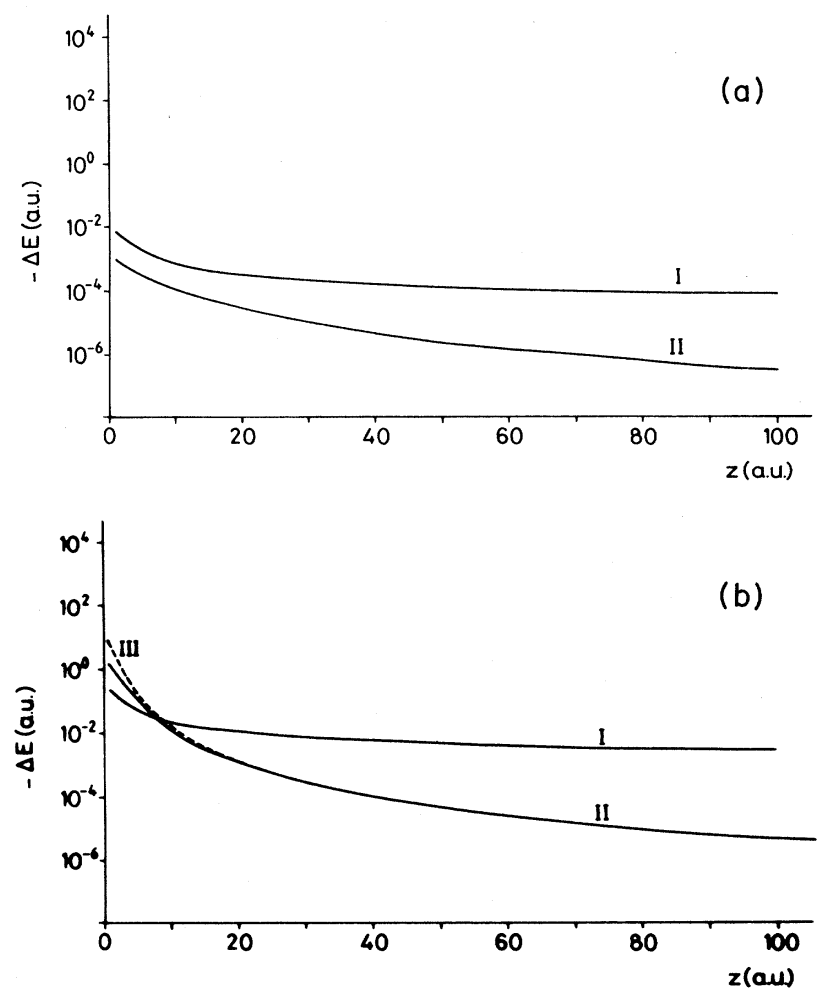

FIG. 2. Static electron self-energy as a function of the electron-planar-surface distance. Curve $I$ is the classical image potential, curve II is the full dielectric calculation of the ripplon contribution, and curve III is the approximate calculation of the ripplon contribution (see text); (a) for helium, $q_{c}=0.10$ a.u., (b) for water, $q_{c}=1.11$ a.u. The values of the parameters used for each liquid are $d=22.75$ a.u., $\sigma=2.244 \times 10^{-7}$ a.u., and $\epsilon=1.057$ for helium and $d=162.504$ a.u., $\sigma=4.673 \times 10^{-5}$ a.u., and $\epsilon=78.1$ for water; $g=1.083 \times 10^{-22}$ a.u.

\section{STATIC CHARGE RIPPLON INTERACTION: SPHERICAL LIQUID SURFACE}

We repeat the procedure followed in the planar case and we obtain the coupling coefficients for ripplon charge interaction for an electron in the $z$ axis. In the Appendix are given the coefficients corresponding to the case in which the electron lies in an arbitrary position.

\section{A. Interaction Hamiltonian}

We consider a spherical drop of radius $R$ centered at the origin of coordinates and we express by $\zeta(\theta, \varphi)$ the surface normal displacement from its nonperturbed position at $R$ [see Fig. 1(b)]. Throughout this section spherical coordinates $(r, \theta, \varphi)$ will be used. We reformulate the steps followed in Sec. II to obtain the adequate expressions for the spherical liquid drop and obtain the expression of the image potential energy $U(z)$ felt by the charge $e$ lying in the $z$ axis (see Appendix): 


$$
\begin{aligned}
U_{\text {rip }}(z)=\frac{e^{2}}{\sqrt{4 \pi}} \frac{\epsilon-1}{(z+R)^{2}} \sum_{l} u_{l 0} \sqrt{2 l+1} \sum_{q} & \frac{q+1}{\epsilon q+q+1} \\
\times\left[q\left[\frac{R}{z+R}\right]^{2 q+1}\right. & \\
& \left.+\frac{2 q+1}{2 l+1} \sum_{l^{\prime}}\left[\frac{R}{z+R}\right]^{l^{\prime}+q} \frac{l^{\prime} \beta}{\left(l^{\prime}+q+2\right)\left(\epsilon l^{\prime}+l^{\prime}+1\right)} C_{0}^{2}\left(l^{\prime} q l ; 000\right)\right],
\end{aligned}
$$

where

$$
\beta=-\left(2 \epsilon q l^{\prime}+2 l^{\prime 2}+3 l^{\prime}+\epsilon q+1\right) .
$$

$z$ is the mean distance from the charge to the spherical surface, $u_{l m}$ are the expansion coefficients of the surface displacement in spherical harmonics:

$$
\zeta(\theta, \phi)=\sum_{l m} u_{l m} Y_{l m}(\theta, \phi),
$$

and $C_{0}$ are Clebsh-Gordan coefficients. ${ }^{23}$

The first term on the right-hand side of Eq. (17) comes from the first-order term of the Taylor expansion of the classical image potential around $\xi=r-(R+\zeta)$. The coefficients $u_{l m}$ have been previously calculated in Ref. 15 in terms of the ripplon creation and annihilation operators and are given $b^{24}$

$$
u_{l m}=\left(\frac{\hbar l}{2 R^{3} d w_{l}}\right)^{1 / 2}\left[(-1)^{m} b_{l-m}^{\dagger}+b_{l m}\right] \text {, }
$$

where

$$
w_{l}^{2}=l(l-1)(l+2) \sigma /\left(d R^{3}\right)
$$

is the ripplon oscillation frequency. ${ }^{20}$ The angular momentum $l$ has to be greater than one; the lowest two modes are excluded by the requirement of mass conservation $(l=0)$, i.e., radial oscillations are forbidden for noncompressible fluids, and by fixing the center of mass of the drop at the origin of the coordinate system $(l=1)$, with which a translation motion of the drop as a whole is not allowed. The effect of a small but finite compressibility of the liquid on the dispersion relation for ripplons has been studied by Ogale et al. $^{25}$

From Eqs. (17) and (20) we obtain the interaction Hamiltonian

$$
H^{1}=\sum_{i=1}^{2} \sum_{l} \alpha_{i}(l, z) \delta_{m, 0}\left[(-1)^{m} b_{l-m}^{\dagger}+b_{l m}\right],
$$

where the coupling coefficients are

$\alpha_{1}=\frac{e^{2}}{\sqrt{4 \pi}}\left(\frac{l(2 l+1)}{2 R^{3} d w_{l}}\right)^{1 / 2} \frac{\epsilon-1}{(z+R)^{2}} \sum_{q} \frac{q(q+1)}{\epsilon q+q+1}\left(\frac{R}{z+R}\right)^{2 q+1}$,
$\left.\alpha_{2}=\frac{e^{2}}{\sqrt{4 \pi}}\left(\frac{l}{2 R^{3} d w_{l}}\right]^{1 / 2} \frac{\epsilon-1}{(z+R)^{2}} \sum_{q} \frac{(q+1)(2 q+1)}{\epsilon q+q+1} \sum_{l^{\prime}} \int \frac{R}{z+R}\right]^{l^{\prime}+q} \frac{l^{\prime} \beta C_{0}^{2}\left(l^{\prime} q l ; 000\right)}{\left(l^{\prime}+q+2\right)\left(\epsilon l^{\prime}+l^{\prime}+1\right)}, l^{\prime}+q+1$ even.

\section{B. Self-energy}

In terms of the coupling coefficients, Eqs. (23), the self-energy of a point charge at a distance $z$ from the spherical surface is

$$
\Delta E(z)=-\sum_{l m}\left|\alpha_{1}+\alpha_{2}\right|^{2} \frac{1}{\hbar w_{l}} .
$$

In Fig. 3 we show numerical results for the self-energy as a function of $u=(z+R) / R$ for helium [Fig. 3(a)] and water for the static [Fig. 3(b)] and optical [Fig. 3(c)] dielectric constants. We take $R=100$ a.u. in all the calculations. To check the applicability of the perturbation approximation we have compared in each case the ripplon contribution to the self-energy with the classical self-energy of a charge in front of a flat sphere i.e.,

$U_{0}(z)=\frac{1}{z+R} \frac{1-\epsilon}{2} \sum_{l} \frac{l}{\epsilon l+l+1}\left(\frac{R}{z+R}\right)^{2 l+1}$.
From Fig. 3 we infer that the ripplon interaction is a perturbation of less than $10 \%$ of the self-energy for distances $z$ larger than 25 a.u. $(u=1.25)$. It must be realized nevertheless, that the ratio between the ripplon contribution and the self-energy of an electron in front of a spherical flat surface depends on the sphere radius, as can be easily shown. Indeed from Eqs. (23)-(25) one can see that the quantity

$$
\Delta E(z)(z+R)^{4}
$$

is independent of the radius. This allows us to obtain the ripplon electron self-energy for an arbitrary radius:

$$
[\Delta E(z)]_{R_{1}}=[\Delta E(z)]_{R_{2}}\left(\frac{R_{2}}{R_{1}}\right)^{4} .
$$

If we expression the self energy of an electron in front of a flat sphere by $\Delta E_{0}(z)$ the quantity that is independent of the radius is now 


$$
\Delta E_{0}(z)(z+R) .
$$

Thus, for different radii we obtain the relation

$$
\left[\Delta E_{0}(z)\right]_{R_{1}}=\left[\Delta E_{0}(z)\right]_{R_{2}} \frac{R_{2}}{R_{1}},
$$

and therefore the ratio between the ripplon and flat surface contributions depends on the radius.

For comparison, we have also calculated the selfenergy using the $r^{-4}$ potential integrated through the volume of the sphere and have obtained for the ripplon contribution
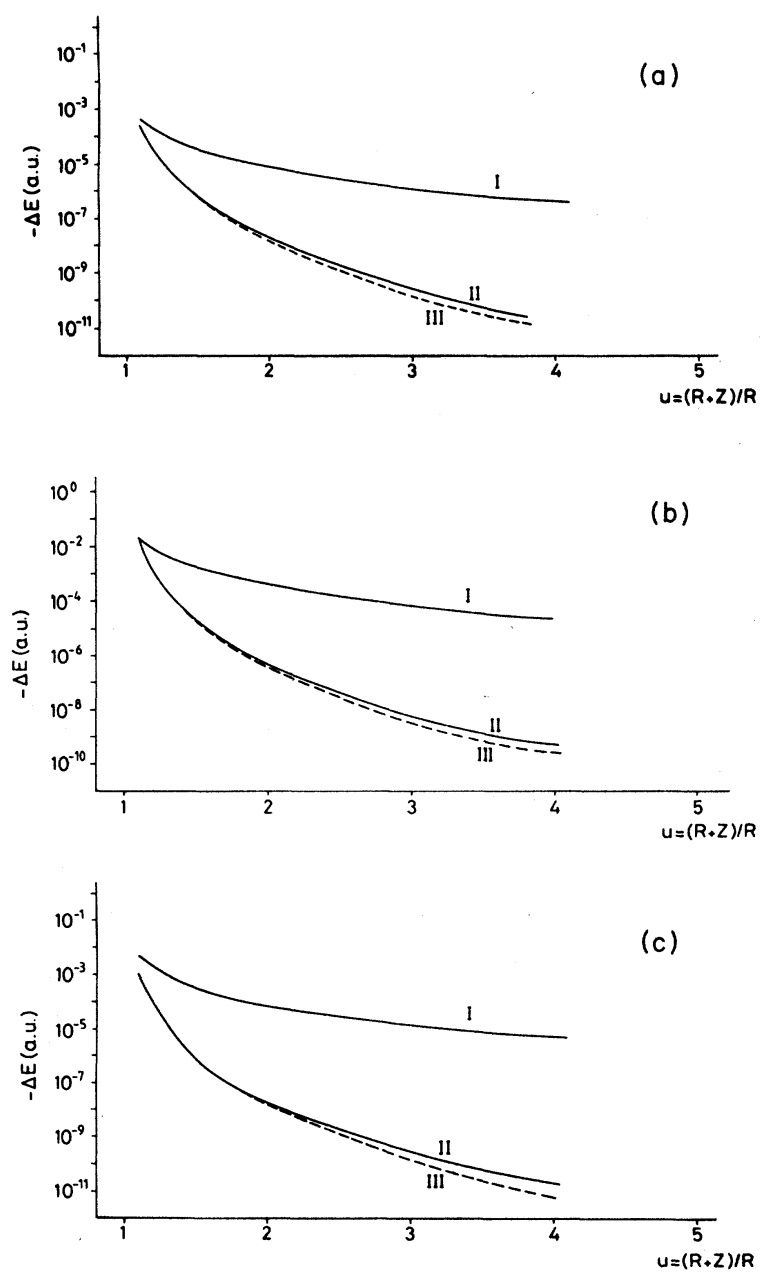

FIG. 3. Self-energy of an electron near a liquid drop as a function of $u=(z+R) / R$. Curve $I$ is the classical image potential, curve II is the full dielectric calculation of the ripplon contribution to the self-energy, and curve III is the same as curve II but obtained from the approximate calculation; (a) for helium, $q_{c}=0.26 R$, (b) for water $q_{c}=1.11 R, \epsilon=78.1$, and (c) for water $q_{c}=1.11 R, \epsilon=1.76$. All other parameters are the same as in Fig. 2. $R=100$ a.u. in all the calculations.

$$
\begin{aligned}
U_{\text {rip }}(z)=\frac{n \alpha}{(z+R)^{2}} \sum_{l} u_{l 0} \sqrt{\pi(2 l+1)} & \\
\times & {\left[\sum_{q \geq 1} 2^{2 q-2} \frac{u^{2 q+1}}{\left(1+u^{2}\right)^{2 q}}\right.} \\
& \left.-\sum_{q \geq l} \alpha_{q}^{l} 2^{q} \frac{u^{q+2}}{\left(1+u^{2}\right)^{q+2}}\right],
\end{aligned}
$$

where $n \alpha$ is the polarizability per unit volume, related to the dielectric constant by

$$
\epsilon=(8 \pi n \alpha+3) /(3-4 \pi n \alpha),
$$

and where

$$
\begin{aligned}
& \alpha_{q}^{l}=\frac{(q+1) q(q-1) \cdots(q-l+2)}{(q+l+1)(q+l-1) \cdots(q-l+3)}, \\
& l+q \text { even, } l \geq 2 .
\end{aligned}
$$

We see from Fig. 3 that the alternative method yields results that worsen when the distance from the surface increases and that this behavior is very similar for polar and nonpolar liquids. This seems to indicate that there are screening effects associated with the spherical geometry which are not included in the approximate calculation and that these screening effects seem to be far more important than the effect of the permanent dipole moment of polar liquids.

The summation over $l$ has a cutoff given by

$$
l_{c}=q_{c} R
$$

where $q_{c}$ is the cutoff used in the planar surface case for the same material at the same temperature.

\section{DYNAMICAL CHARGE RIPPLON INTERACTION: PLANAR LIQUID SURFACE}

In order to calculate the stopping power corresponding to an electron moving with velocity $\bar{v}$ parallel and external to a planar surface we substitute the electronic twodimensional position vector $\bar{\rho}$ by $\bar{v} t$ in the interaction Hamiltonian $H^{\prime}$, Eq. (8). From this time-dependent Hamiltonian the $q$ mode excitation probability at time $t$ is given by

$$
P_{\overline{\mathbf{q}}}(t)=\frac{1}{\hbar^{2}}\left|\int_{-\infty}^{t} d t^{\prime} e^{i w_{q} t^{\prime}}\left\langle\overline{\mathbf{q}}\left|H^{\prime}\left(t^{\prime}\right)\right| 0\right\rangle\right|^{2},
$$

and the stopping power by

$$
\frac{d W}{d x}=\frac{\Omega}{(2 \pi)^{2}} \int d^{2} q \frac{\hbar w_{q}}{v} \gamma_{\bar{q}},
$$

where

$$
\gamma_{\overline{\mathrm{q}}}=\frac{d P_{\overline{\mathrm{q}}}(t)}{d t}
$$

is the rate of the transition from the initial state $|0\rangle$ to the final state $|\bar{q}\rangle$. 
We obtain the following analytic expression for the stopping power:

$$
\frac{d W}{d x}=\sum_{i=1}^{6} S_{i},
$$

where the six terms are given by

$$
\begin{aligned}
& S_{1}=4 D v^{2} \int_{0}^{a} d t \frac{t^{3} K_{1}^{2}(t)}{\sqrt{1-\eta t}}, \\
& S_{2}=D v^{4} \int_{0}^{a} d t \frac{t^{5} k_{0}^{2}(t)}{\sqrt{1-\eta t}}, \\
& S_{3}=\frac{8 D}{3 \eta^{2}} v^{2}\left[2-\left(\eta q_{c} z+2\right)\left(1-\eta q_{c} z\right)^{1 / 2}\right] \\
& S_{4}=4 D v^{3} \int_{0}^{a} d t \frac{t^{4} K_{1}(t) K_{0}(t)}{\sqrt{1-n t}}, \\
& S_{5}=-8 D v^{2} \int_{0}^{a} d t \frac{t^{2} K_{1}(t)}{\sqrt{1-\eta t}}, \\
& S_{6}=-4 D v^{3} \int_{0}^{a} d t \frac{t^{3} K_{0}(t)}{\sqrt{1-\eta t}},
\end{aligned}
$$

where

$$
D=\frac{1}{\left(128 \pi d v^{2} z^{6}\right)}, \quad v=\frac{(\epsilon-1)}{(\epsilon+1)}, \quad a=z q_{c}
$$

and where $\eta=\sigma /\left(d v^{2} z\right)$ and $q_{c}$ is the momentum cutoff (we use atomic units in the above expression).

In Fig. 4(a) we display $d W / d x$ as a function of the distance to the helium surface for an electron moving with velocity $v=85$ a.u. (corresponding to an energy of about $100 \mathrm{keV})$. The alternative calculation using the $r^{-4}$ potential is not depicted because in a logarithmic scale it would be indistinguishable, the differences go from $0.6 \%$ at $z=6$ a.u. to $0.01 \%$ at $z=50$ a.u. The same calculation is shown in Fig. 4(b) for water and in this case the differences with the alternative method are $4.5 \%$ at $z=6$ a.u. and $0.02 \%$ at $z=80$ a.u.

For comparison we show in Fig. 4(b) numerical results for the stopping power of an electron moving over a flat water surface calculated from the expression (in atomic units),

$$
\frac{d W}{d x}=\frac{w_{s}^{2}}{v^{2}} K_{0}\left[\frac{2 w_{s} z}{v}\right],
$$

where $w_{s}=0.546$ a.u. is the surface plasmon frequency for water. From this figure we conclude that the energy lost by ripplon excitations of a classical swift electron over a water surface is negligible. However, energy loss to ripplon excitations become important for lower electron energies, especially around and below the threshold for plasmon excitation.
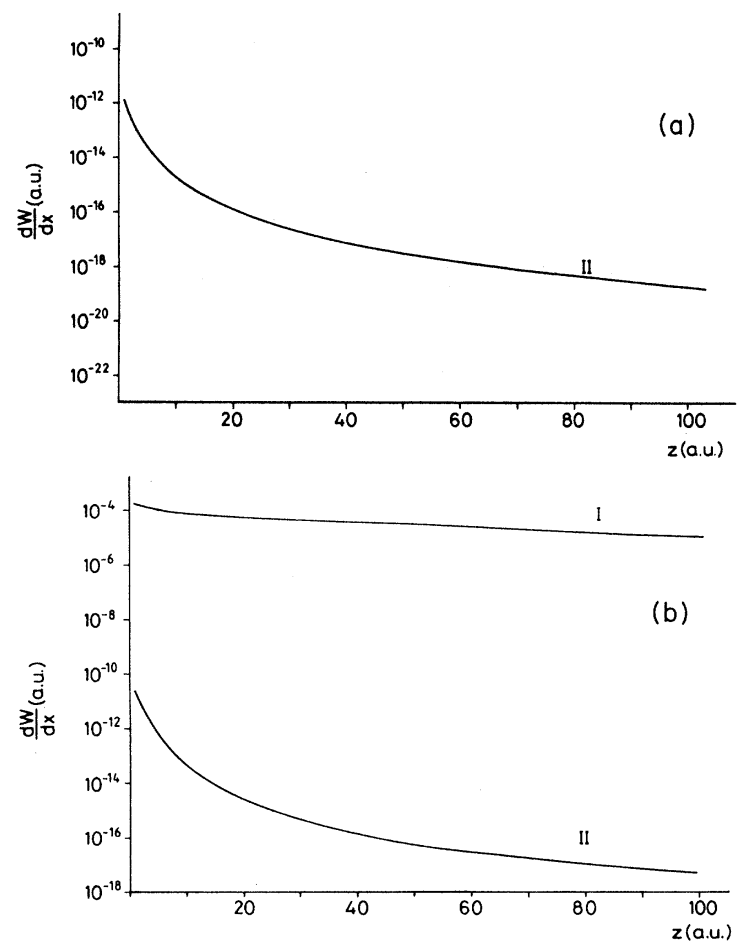

FIG. 4. Stopping power for an electron moving with velocity $v=85$ a.u. parallel to a plane surface. Curve $I$ is the stopping power for a flat surface, curve II is the contribution of the ripplon interaction to the loss of energy; (a) for helium $q_{c}=0.92$ a.u., $\epsilon_{s} \approx \epsilon_{0 p}$ and (b) for water $\epsilon_{0 p}=1.76, q_{c}=1.11$ a.u.

\section{FINAL COMMENTS}

Our calculations have shown that the ripplon contribution to the self-energy of an external electron above a polar liquid is about ten times larger than the one corresponding to an electron above a nonpolar liquid. This result is independent of the geometry considered in this paper [see Figs. 2(a), 2(b), 3(a), and 3(b)].

We also conclude that for polar liquids, in spite of the time consuming calculations, (especially for small values of $u$ in the spherical case), the complete dielectric computation must be performed for the static case.

We have also confirmed the Shikin and Monarkha ${ }^{2}$ results on nonpolar liquids, i.e., the ripplon contribution to the self-energy is a perturbation. However, for polar liquids and short distances the applicability of the first order perturbation method is uncertain (see Figs. 2 and 3).

For the spherical case of a water drop containing a positive ion at its center, Ballester and Antoniewicz ${ }^{11}$ calculated the binding energy and the surface-state wave functions for an electron external to the liquid drop. This electron is attracted both by the classical image potential and by the Coulomb screened interaction with the internal ion. For a water drop of radius $R=5 \AA$ they obtain a binding energy of $1.8 \mathrm{eV}$ for the lowest electronic state located at 2-3 $\AA$ from the surface. For this distance from 
the liquid surface $u=1.4,{ }^{26}$ and from Eq. (27) and Fig. 3(c) we obtain that the ripplon contribution to the selfenergy is about $0.7 \mathrm{eV}$, i.e., about half the binding energy calculated in Ref. 11. This result tells us that the electron in a surface state of a water drop is bound by a stronger force, an important point to be considered in problems like that of the charge-state dependent interaction between liquid drops which induces drop accretion and increases rate of coagulation of aerosols. ${ }^{27}$

Finally, it has been shown that the ripplon contribution to the stopping power for a swift electron running parallel over a planar surface is negligible for high electron energies $(v \gg>1$ a.u.) and the same result should be expected for the spherical case.

\section{ACKNOWLEDGMENTS}

Suggestions and early discussions of this project by Professor R. H. Ritchie are gratefully acknowledged. We wish to thank Professor P. Pascual for his fruitful help in the mathematical development. This work is partly funded by the Spanish Comisión Interministerial de Ciencia y Tecnologia and by the Office of Health and Environmental Research, U.S. Department of Energy, under Contract No. DE-AC-5-840R21400 with Martin Marietta Energy Systems, Inc.

\section{APPENDIX: COUPLING COEFFICIENTS OF THE ELECTRON SPHERICAL SURFACE RIPPLON INTERACTION}

We first calculate the potential $\phi\left(\overline{\mathbf{r}}, \overline{\mathbf{r}}^{\prime}\right)$ created at $\overline{\mathbf{r}}$ by the presence of a spherical dielectric drop of radius $R$ centered at the origin of the coordinate system and an external charge $e$ located in vacuum at $\overline{\mathbf{r}}^{\prime}$. The corresponding Poisson's equations are

$$
\begin{aligned}
& \nabla^{2} \phi\left(\overline{\mathbf{r}}, \overline{\mathbf{r}}^{\prime}\right)=-4 \pi e \delta\left(\overline{\mathbf{r}}-\overline{\mathbf{r}}^{\prime}\right), \quad r \geq R+\zeta(\theta, \varphi), \\
& \nabla^{2} \phi\left(\overline{\mathbf{r}}, \overline{\mathbf{r}}^{\prime}\right)=0, \quad r \leq R+\zeta(\theta, \varphi),
\end{aligned}
$$

where $\zeta(\theta, \varphi)$ is defined in Sec. III. Applying the stan- dard boundary conditions on $\phi$

$$
\begin{aligned}
& \left.\phi\left(\overline{\mathbf{r}}, \overline{\mathbf{r}}^{\prime}\right)\right|_{r=R+\zeta^{-}}=\left.\phi\left(\overline{\mathbf{r}}, \overline{\mathbf{r}}^{\prime}\right)\right|_{r=R+\zeta^{+},} \\
& \left.\epsilon \hat{\mathbf{n}} \cdot \bar{\nabla} \phi\left(\overline{\mathbf{r}}, \overline{\mathbf{r}}^{\prime}\right)\right|_{r=R+\zeta^{-}}=\left.\widehat{\mathbf{n}} \cdot \bar{\nabla} \phi\left(\overline{\mathbf{r}}, \overline{\mathbf{r}}^{\prime}\right)\right|_{r=R+\zeta^{+}},
\end{aligned}
$$

where + and - indicate inside and outside the surface respectively, we obtain

$$
\begin{aligned}
\phi\left(\overline{\mathbf{r}}, \overline{\mathbf{r}}^{\prime}\right)= & e \sum_{l} \frac{r_{<}^{l}}{r_{>}^{l+1}} P_{l}(\cos \Theta) \\
& +e \sum_{l} \frac{1}{\left(r r^{\prime}\right)^{l+1}} \frac{l(1-\epsilon)}{\epsilon l+l+1} R^{2 l+1} P_{l}(\cos \Theta) \\
& +\sum_{l} \frac{A_{l}}{r^{l+1}} P_{l}(\cos \Theta), \quad r, r^{\prime} \geq R+\zeta
\end{aligned}
$$

where $r_{<}\left(r_{>}\right)$denotes the lesser (greater) of $r^{\prime}$ and $r, \hat{\mathbf{n}}$ is the unit vector perpendicular to the surface and is given by

$$
\widehat{\mathbf{n}}=\frac{\overline{\mathbf{n}}}{|\overline{\mathbf{n}}|}=\frac{1}{|\overline{\mathbf{n}}|}\left(1,-\frac{1}{r} \frac{\partial \xi}{\partial \theta},-\frac{1}{r \sin \theta} \frac{\partial \zeta}{\partial \varphi}\right) .
$$

$P_{l}(\cos \Theta)=[4 \pi /(2 l+1)] \sum_{m=-l}^{l} Y_{l m}^{*}\left(\Omega^{\prime}\right) Y_{l m}(\Omega) \quad$ are Legendre polynomials and $Y_{l m}(\Omega)$ are the spherical harmonics. The argument in the Legendre polynomials is $\cos \theta=\cos \theta \cos \theta^{\prime}+\sin \theta \sin \theta^{\prime} \cos \left(\varphi-\varphi^{\prime}\right)$. The angular coordinates $\Omega$ and $\Omega^{\prime}$ refer, respectively, to the angular positions where the potential is calculated and where the external charge is located. $A_{l}$ is a function of $\overline{\mathbf{r}}^{\prime}$ and the displacement $\zeta$ [we shall only retain terms up to $O(\zeta)$ in what follows].

The first term in the right-hand side of Eq. (A3) is the bare Coulomb potential, the second term is the potential produced by the nonrippled surface, and the third one is that generated by the rippling surface.

If we remove the bare Coulomb term from $\phi$ and calculate the force on a test charge $e_{0}$ at $\overline{\mathbf{r}}$ along the radial direction $\left(F_{r}=-e_{0} \partial \phi\left(\overline{\mathbf{r}}, \overline{\mathbf{r}}^{\prime}\right) / \partial \overline{\mathbf{r}}\right)$ we obtain the image potential energy $U(\overline{\mathbf{r}})$ felt by the charge $e$ after identifying the test charge $e_{0}$ and the charge $e$ at $\overline{\mathbf{r}}^{\prime}\left(\overline{\mathbf{r}}=\overline{\mathbf{r}}^{\prime}, e=e_{0}\right)$ and up to the first-order ripplon contribution we obtain

$$
\begin{aligned}
U_{\text {rip }}= & -e^{2} \sum_{l} \frac{l(l+1)}{\epsilon l+l+1}(1-\epsilon) \frac{R^{2 l+1}}{r^{2 l+3}} \sum_{l^{\prime} m^{\prime}} u_{l^{\prime} m^{\prime}} Y_{l^{\prime} m^{\prime}} \\
+ & +e^{2} \sum_{q} \frac{(2 q+1)(q+1)}{\epsilon q+q+1} \sum_{l} \frac{R^{l+q}}{r^{l+q+2}} \frac{1-\epsilon}{(l+q+2)(\epsilon l+l+1)} \\
& \times\left[l \beta \sum_{l^{\prime} m^{\prime}} u_{l^{\prime} m^{\prime}} \frac{Y_{l^{\prime} m^{\prime}}}{2 l^{\prime}+1} C_{0}^{2}\left(l q l^{\prime} ; 000\right)-\frac{2 l+1}{4}\left(d_{u}+d_{\varphi}\right)\right],
\end{aligned}
$$

where $\beta$ is given in Eq. (18), $u_{l m}$ in Eq. (20) and

$$
\begin{aligned}
d_{u}=\sum_{l^{\prime} m^{\prime}} \sum_{m=-l m^{\prime \prime}=-q}^{l} \sum^{q} & {\left[\frac{4 \pi}{(2 l+1)(2 q+1)}\right]^{1 / 2} Y_{l m}^{*} Y_{q m^{\prime \prime}}^{*} \frac{(-1)^{m^{\prime}}}{2 l+1}\left(u_{l^{\prime}-m^{\prime}}+u_{l^{\prime} m^{\prime}}\right) } \\
\times & {\left[N_{l^{\prime} m^{\prime}} \sum_{r \leq l^{\prime}-1}\left\{\left(l^{2}-m^{2}\right)^{1 / 2}(l+1) C_{-} C_{-}^{0}-\left[(l+1)^{2}-m^{2}\right]^{1 / 2} l C_{+} C_{+}^{0}\right\} \frac{(2 r+1)^{1 / 2}}{N_{r m^{\prime}}}\right.} \\
& \left.\quad-\frac{1}{N_{l^{\prime} m^{\prime}}} \sum_{r \geq l^{\prime}+1}\left\{\left(l^{2}-m^{2}\right)^{1 / 2}(l+1) C_{-} C_{-}^{0}-\left[(l+1)^{2}-m^{2}\right]^{1 / 2} l C_{+} C_{+}^{0}\right\} N_{r m^{\prime}} \frac{2 l^{\prime}+1}{(2 r+1)^{1 / 2}}\right],
\end{aligned}
$$


where $r+l^{\prime}+1$ is even, $l+q+r+1$ is even, and $m^{\prime}=m+m^{\prime \prime}$,

$$
\begin{aligned}
& d_{\varphi}=\sum_{l^{\prime} m^{\prime}>0} \sum_{m=-l m^{\prime \prime}=-q}^{l} \sum_{m}^{q}(-1)^{m^{\prime}}\left(u_{l^{\prime}-m^{\prime}}-u_{l^{\prime} m^{\prime}}\right) m\left[\frac{4 \pi}{(2 l+1)(2 q+1)}\right]^{1 / 2} Y_{l m}^{*} Y_{q m^{\prime \prime}}^{*} \\
& \times\left[N_{l^{\prime} m^{\prime}} \sum_{r \leq l^{\prime}} \frac{(2 r+1)^{1 / 2}}{N_{r m^{\prime}}} C C_{0}+\frac{2 l^{\prime}+1}{N_{l^{\prime} m^{\prime}}} \sum_{r \geq l^{\prime}+2} \frac{N_{r m^{\prime}}}{(2 r+1)^{1 / 2}} C C_{0}\right],
\end{aligned}
$$

where $r+l^{\prime}$ is even, $l+q+r$ is even, and $m^{\prime}=m+m^{\prime \prime}$,

$$
\begin{aligned}
& C_{\mp}=C\left(l \mp 1, q, r ; m m^{\prime \prime} n\right), \\
& C_{\mp}^{0}=C(l \mp 1, q, r ; 000)
\end{aligned}
$$

are Clebsch-Gordan coefficients, and

$$
N_{l m}=\left[\frac{2 l+1}{4 \pi} \frac{(l-m) !}{(l+m) !}\right]^{1 / 2}
$$

Using Eq. (20) we obtain the interaction Hamiltonian given by

$$
H^{\prime}=\sum_{l m}\left(\alpha_{1}+\alpha_{2}\right)\left[(-1)^{m} b_{l-m}^{\dagger}+b_{l m}\right]+\sum_{l m>0} \alpha_{3}\left[(-1)^{m} b_{l m}^{\dagger}+b_{l-m}+(-1)^{m} b_{l-m}^{\dagger}+b_{l m}\right],
$$

with the coupling coefficients,

$$
\begin{aligned}
& \alpha_{1}=B Y_{l m} \sum_{q} \frac{q(q+1)}{\epsilon q+q+1}(\epsilon-1) \frac{R^{2 q+1}}{r^{2 q+3}}, \\
& \alpha_{2}=B \frac{Y_{l m}}{2 l+1} \sum_{l^{\prime} q} \frac{(2 q+1)(q+1)}{\epsilon q+q+1} \frac{R^{l^{\prime}+q}}{r^{l^{\prime}+q+2}} \frac{l^{\prime} \beta(1-\epsilon)}{\left(l^{\prime}+q+2\right)\left(\epsilon l^{\prime}+l^{\prime}+1\right)} C_{0}^{2}\left(l^{\prime} q l ; 000\right), l^{\prime}+q+l \text { even }, \\
& \alpha_{3}=B \sum_{l^{\prime} q} \frac{(2 q+1)(q+1)}{\epsilon q+q+1} \frac{R^{l^{\prime}+q}}{r^{l^{\prime}+q+2}} \frac{(\epsilon-1)\left(1-\delta_{m 0}\right)}{4\left(l^{\prime}+q+2\right)\left(\epsilon l^{\prime}+l^{\prime}+1\right)} \\
& \times \sum_{m^{\prime}=-l^{\prime}}^{l^{\prime}} \sum_{m^{\prime \prime}=-q}^{q}\left(\frac{4 \pi}{\left(2 l^{\prime}+1\right)(2 q+1)}\right]^{1 / 2} Y_{l^{\prime} m^{\prime}}^{*} Y_{q m^{\prime \prime}}^{*}(-1)^{m}\left[N_{l m} \sum_{r \leq l-1} \mu \frac{(2 r+1)^{1 / 2}}{N_{r m}}\right. \\
& \left.-\frac{1}{N_{l m}} \sum_{r \geq l+1} \mu N_{r m} \frac{2 l+1}{(2 r+1)^{1 / 2}}\right] \text {, }
\end{aligned}
$$

where

$$
\begin{aligned}
& \mu=\left\{\left(l^{\prime 2}-m^{\prime 2}\right)^{1 / 2}\left(l^{\prime}+1\right) C_{-} C_{-}^{0}-\left[\left(l^{\prime}+1\right)^{2}-m^{\prime 2}\right]^{1 / 2} l^{\prime} C_{+} C_{+}^{0}\right\}, \\
& C_{\mp}=C\left(l^{\prime} \mp 1, q, r ; m^{\prime} m^{\prime \prime} n\right) \text { and } B=\left[\hbar l /\left(2 R^{3} d w_{l}\right)\right]^{1 / 2} .
\end{aligned}
$$

${ }^{1}$ T. R. Brown and C. C. Grimes, Phys. Rev. Lett. 29, 1233 (1974).

${ }^{2}$ V. B. Shikin and Yu. P. Monarkha, J. Low Temp. Phys. 16, 193 (1974).

${ }^{3}$ M. W. Cole, Rev. Mod. Phys. 46, 451 (1974).

${ }^{4}$ D. S. Fisher, B. I. Halperin, and P. M. Platzman, Phys. Rev. Lett. 42, 798 (1979).

${ }^{5}$ K. Kono, Surf. Sci. 170, 75 (1986).

${ }^{6}$ A. G. Equiluz, A. A. Maradudin, and R. J. Elliott, Phys. Rev. B 24, 197 (1981).

${ }^{7}$ Proceedings of the 4th International Conference on Electronic Properties of Two-Dimensional Systems New London, New Hampshire, 1981 [Surf. Sci. 113, 1 (1982)].

${ }^{8}$ M. Armbruster, H. Haberland, and H. G. Schindler, Phys. Rev. Lett. 47, 323 (1981).

${ }^{9}$ R. N. Barnett, U. Landman, C. L. Cleveland, and J. Jortner,
Phys. Rev. Lett. 59, 811 (1987).

${ }^{10}$ A. Wallqvist, D. Thirumalai, and B. J. Berne, J. Chem. Phys. 85, 1583 (1986).

${ }^{11}$ J. L. Ballester and P. R. Antoniewicz, J. Chem. Phys. 85, 5204 (1986).

${ }^{12}$ C. C. Grimes and G. Adams, Phys. Rev. Lett. 36, 145 (1976); 42, 795 (1979).

${ }^{13}$ P. M. Echenique and J. B. Pendry, J. Phys. C 9, 3183 (1976).

${ }^{14}$ T. S. Rahman and A. A. Maradudin, Phys. Rev. B 21, 504 (1980).

${ }^{15}$ A. Gras-Marti and R. H. Ritchie, Phys. Rev. B 31, 2649 (1985).

${ }^{16}$ M. W. Cole, Phys. Rev. B 2, 4239 (1970).

${ }^{17}$ A. G. Eguiluz, A. A. Maradudin, and R. J. Elliott, in Ab Initio Calculation of Phonon Spectra, edited by J. T. Devreese (Plenum, New York, 1983), p. 269. 
${ }^{18}$ T. S. Rahman and D. L. Mills, Phys. Rev. B 21, 1432 (1980).

${ }^{19}$ Handbook of Mathematical Functions, edited by $\mathbf{M}$. Abramowitz and I. Stegun (Dover, New York, 1965), p. 374.

${ }^{20}$ L. D. Landau and E. M. Lifshitz, Fluid Mechanics (Pergamon, Oxford, 1959), pp. 238 and 240.

${ }^{21}$ M. W. Cole and M. H. Cohen, Phys. Rev. Lett. 23, 1236 (1969).

${ }^{22}$ R. Loudon, in Surface Excitations, edited by V. M. Agranowich and R. Loudon (North-Holland, Amsterdam, 1984), p. 589.

${ }^{23}$ D. M. Brink and G. R. Satchler, Angular Momentum (Clarendon, Oxford, 1968).
${ }^{24}$ Note that a misprint appearing in Eq. (A18) in Ref. 15 has been corrected in Eq. (20).

${ }^{25}$ S. B. Ogale, P. V. Panat, and J. Mahanty, Physica 110A, 593 (1982).

${ }^{26}$ Note that it is easily shown from Eq. (20) that the mean value of the surface displacement for capillary waves in the watervapor interface is always smaller than 0.07 a.u. or $u=1.0007$, i.e., the liquid does not wet an electron in a surface state. This holds for helium too.

${ }^{27}$ W. H. Marlow, in Aerosol Microphysics I: Partial Interactions, edited by W. H. Marlow (Springer, New York, 1980). 